Notre Dame Journal of Formal Logic Volume 22, Number 3, July 1981

\title{
Quine on an Alleged Non Sequitur
}

\author{
ALEX BLUM
}

In articles by Wiredu [4] and, more recently, Merrill [1], Quine is taken to task for an alleged non sequitur in "The Problem of Interpreting Modal Logic" [2]. My object is to correct this misimpression.*

Quine argues that if we assume:

(ii) An existential quantification holds if there is a constant whose substitution for the variable of quantification would render the matrix true ([2], p. 271)

then given the truth of both:

(1) Morning Star $C$ Evening Star $\square$ (Morning Star $C$ Morning Star)

(2) Evening Star $C$ Evening Star $\sim \square$ (Evening Star $C$ Morning Star)

"where ' $C$ ' (for 'congruence') . . . is used to express the relation which Venus, the Evening Star and the Morning Star, e.g., bear to themselves and, . . . to one another" ([2], p. 272), it follows that both:

(3) $(\exists x)(x C$ Evening Star $\cdot \square(x C$ Morning Star $))$

(4) $(\exists x)(x C$ Evening Star $\sim \square(x C$ Morning Star $))$

are true. And given that the matrices quantified in (3) and (4) are contraries, "there must be at least two objects $x$ such that $x C$ Evening Star" ([2], p. 272).

Wiredu contends that all that follows from (3) and (4) on the substitutional interpretation of quantification that Quine in (ii) is allegedly committed to, is that "there must be at least two constants such that their substitution in ' $x C$ Evening Star' renders the matrix true" ([4], p. 188). And in a similar vein, Merrill writes: "What Quine is entitled to infer in the light of (ii) . . is

$(* * *)$ so there must be at least two objects (names) $x$ such that the sentences ${ }^{\top} x C$ Evening $\left.\operatorname{Star}\right]^{-}$is true". ([1], pp. 614-615)

\footnotetext{
*I am indebted to David Widderker for helpful conversations on the issues in this paper, and to the referee of this Journal for his corrections.
} 
But clearly the Wiredu-Merrill criticism is warranted only if we take (ii) to be a statement of the substitutional interpretation of quantification, which it is not. Quine takes (ii) as a sufficient condition for the truth of an existential quantifier and states immediately after:

This is at best a partial criterion (both in modal and non-modal logic), because of unnameable objects; an unnameable object might satisfy the matrix though there be no constant expression whose substitution for the variable would yield a truth. But let us adopt (ii) as a partial criterion (sufficient condition) and see how it fares. ([2], p. 271)

Quine thus takes (1) and (2) to imply, on the basis of (ii), (3) and (4) read objectually. The substitutional reading of quantification is considered by Quine for the first time and with some alarm in his much later [3].

\section{REFERENCES}

[1] Merrill, A. H., "On an enduring non sequitur of Quine's," Notre Dame Journal of Formal Logic, vol. 18, no. 4 (1977), pp. 613-615.

[2] Quine, W. V. O., "The problem of interpreting modal logic," The Journal of Symbolic Logic, vol. 12, 1947. Reprinted in Irving M. Copi and James A. Gould, eds., Contemporary Readings in Logical Theory, Macmillan, New York, 1967. All page references are to this reprint.

[3] Quine, W. V. O., "Reply to Professor Marcus," Synthese, vol. 13, no. 4 (1961), pp. 323-330.

[4] Wiredu, J. E., "A note on modal quantification, ontology, and the indenumerably infinite," Analysis, vol. 32, no. 6 (1972), pp. 187-191.

Department of Philosophy

Bar-Ilan University

Ramat-Gan, Israel 